\title{
AN UNUSUAL CONSTRUCTION OF THE FOURIER TRANSFORM
}

\author{
David Malone
}

\section{Introduction}

The usual construction of the Fourier transform involves working on $L^{1}(\mathbb{R})$ (eg. [1]) or the Schwartz Class $\mathcal{S}$ of rapidly decreasing $C^{\infty}$ functions (eg. [2]). The Fourier transform is then extended onto $L^{2}(\mathbb{R})$ by taking limits as both $L^{1}(\mathbb{R}) \cap L^{2}(\mathbb{R})$ and $\mathcal{S}$ are dense in $L^{2}(\mathbb{R})$.

I'd like to present an unusual construction of the Fourier transform in which we use its translation and dilation properties:

- if $g(x)=f(x+\alpha)$ then $\hat{g}(\omega)=e^{i \alpha \omega} \hat{f}(\omega)$,

- if $g(x)=f(\lambda x)$ then $\hat{g}(\omega)=1 /|\lambda| \hat{f}(\omega / \lambda)$.

This construction is in the spirit of the "multiresolution analysis" structure [3] which is used to build discrete wavelet bases [4]. However, if you don't know anything about this structure the construction is still surprisingly straightforward.

\section{The Definition}

I'll be using both $\hat{f}$ and $\mathcal{F} f$ to denote the Fourier transform of $f$. For translation and dilation I'll write:

- $\left(\mathcal{T}_{\alpha} f\right)(x):=f(x+\alpha)$ for any $\alpha \in \mathbb{R}$,

- $(\mathcal{D} \lambda f)(x):=f(\lambda x)$ for any $\lambda \in \mathbb{R} \backslash\{0\}$,

- $(\mathcal{R} \alpha f)(x):=e^{i \alpha x} f(x)$ for any $\alpha \in \mathbb{R}$.

This allows me to write the translation and dilation properties as

$$
\mathcal{F} \mathcal{T}_{\alpha} f=\mathcal{R}_{\alpha} \mathcal{F} f
$$

and 


$$
\mathcal{F} \mathcal{D}_{\lambda} f=\frac{1}{|\lambda|} \mathcal{D}_{\frac{1}{\lambda}} \mathcal{F} f .
$$

Now to define $\mathcal{F}$, we do the following:

1. for the characteristic function of $[0,1)$ we define

$$
\mathcal{F}\left(\chi_{[0,1)}\right)=\frac{1-e^{-i \omega}}{i \omega},
$$

2. we extend $\mathcal{F}$ to any $\chi_{[n, n+1)}$ by using the translation rule

$$
\mathcal{F} \mathcal{T}_{n} f=\mathcal{R}_{n} \mathcal{F} f
$$

for all $n \in \mathbb{Z}$,

3. we further extend $\mathcal{F}$ to functions of the form $\chi_{\left[\lambda^{-1} n, \lambda^{-1}(n+1)\right)}$ by using the dilation rule

$$
\mathcal{F D}_{\lambda} f=\frac{1}{|\lambda|} \mathcal{D}_{\frac{1}{\lambda}} \mathcal{F} f
$$

for all $\lambda \in 2^{\mathbb{Z}}$,

4. finally we extend $\mathcal{F}$ to the linear span of these by assuming that $\mathcal{F}$ is linear.

If this process works, we have defined $\mathcal{F}$ on $D$, the set of dyadic step functions. These are just the simple functions whose jumps occur at $n / 2^{m}$ where $n, m \in \mathbb{Z}$. It is easy to construct a well defined function which has these properties. In fact the definition spells out a formula:

$$
\begin{aligned}
f(x) & =\sum_{r=-R}^{R} a_{r} \chi_{[0,1)}\left(2^{j} x-r\right) \Rightarrow \\
\mathcal{F} f(\omega) & =\frac{1-e^{-i \omega / 2^{j}}}{i \omega} \sum_{r=-R}^{R} a_{r} e^{-i r \omega / 2^{j}} .
\end{aligned}
$$

Our aim was to produce $\mathcal{F}$ on $L^{2}(\mathbb{R})$. Given that the set of simple functions is dense in $L^{2}(\mathbb{R})$ it is clear that $D$ is also dense in 
$L^{2}(\mathbb{R})$. So, if we can show that this function $\mathcal{F}$ we have defined is continuous in the $L^{2}(\mathbb{R})$ norm then we can extend $\mathcal{F}$ to all of $L^{2}(\mathbb{R})$.

This turns out to be surprisingly straight forward. Taking

$$
f(x)=\sum_{r=-R}^{R} a_{r} \chi_{[0,1)}\left(2^{j} x-r\right)
$$

we see that $\|f\|_{2}^{2}=\sum_{r=-R}^{R}\left|a_{r}\right|^{2} / 2^{j}$.

Now we have to find $\|\mathcal{F} f\|_{2}^{2}$. Using our formula above and the definition of the norm:

$$
\begin{aligned}
& \|\mathcal{F}(f)\|_{2}^{2}= \\
& \int_{-\infty}^{\infty}\left|\frac{1-e^{-i \frac{\omega}{2^{j}}}}{i \omega}\right|^{2}\left(\sum_{k=-N}^{N} a_{k} e^{-i k \frac{\omega}{2^{j}}}\right)\left(\sum_{l=-N}^{N} \overline{a_{l}} e^{i l \frac{\omega}{2^{j}}}\right) d \omega= \\
& \int_{-\infty}^{\infty} \frac{2\left(1-\cos \frac{\omega}{2^{j}}\right)}{\omega^{2}}\left[\left(\sum_{k=-N}^{N}\left|a_{k}\right|^{2}\right)+\left(\sum_{k \neq l} a_{k} \overline{a_{l}} e^{-i(k-l) \frac{\omega}{2^{j}}}\right)\right] d \omega
\end{aligned}
$$

So we need to evaluate:

$$
\int_{-\infty}^{\infty} \frac{2(1-\cos \omega)}{\omega^{2}} e^{i r \omega} d \omega
$$

for $r \in \mathbb{Z}$. This is an easy piece of contour integration, giving $2 \pi$ if $r=0$ and zero otherwise. Filling this in we see:

$$
\|\mathcal{F} f\|_{2}^{2}=\sum_{r=-R}^{R}\left|a_{r}\right|^{2} / 2^{j} 2 \pi=2 \pi\|f\|_{2}^{2}
$$

So, not only is $\mathcal{F}$ continuous but it just scales the norm. This means that we may extend $\mathcal{F}$ to a continuous map from $L^{2}(\mathbb{R}) \rightarrow$ $L^{2}(\mathbb{R})$ which preserves the inner product:

$$
(f, g)=2 \pi(\mathcal{F} f, \mathcal{F} g) .
$$




\section{What now?}

Note that we could show that $\mathcal{F}$ as defined on $D$ also extends to a continuous map $\mathcal{F}: L^{1}(\mathbb{R}) \rightarrow L^{\infty}(\mathbb{R})$ by examining the $L^{1}(\mathbb{R})$ norm of $f$ and the $L^{\infty}(\mathbb{R})$ norm of of $\mathcal{F} f$. This might motivate us to try to get the usual integral formula for the Fourier transform back again.

This can be done for $f \in D$ by first considering $f$ as a function with steps of width $2^{-j}$, and then splitting each step in half to get the same function written in terms of steps of width $2^{-(j+1)}$. This turns our formula for $\mathcal{F}$ into a Riemann sum for the integral:

$$
(\mathcal{F} f)(\omega)=\int f(x) e^{-i \omega x} d x \quad f \in D .
$$

This can naturally be extended to suitable sets larger than $D$.

By looking at the dilation and translation relations carefully (or by using the integral formula) we get extended translation and dilation, this time for all $f \in L^{2}(\mathbb{R}), \alpha \in \mathbb{R}$ and $\lambda \in \mathbb{R} \backslash\{0\}$ :

$$
\begin{aligned}
& \text { - } \mathcal{F} \mathcal{T}_{\alpha} f=\mathcal{R}_{\alpha} \mathcal{F} f, \\
& \text { - } \mathcal{F} \mathcal{D}_{\lambda} f=\frac{1}{|\lambda|} \mathcal{D} \frac{1}{\lambda} \mathcal{F} f, \\
& \text { - } \mathcal{F} \mathcal{R}_{\alpha} f=\mathcal{T}_{-\alpha} \mathcal{F} f .
\end{aligned}
$$

This provides us with a neat way to show that $\mathcal{F}$ is invertible on $L^{2}(\mathbb{R})$. Suppose we defined $\mathcal{G}$ with the translation and dilation properties we expect of $\mathcal{F}^{-1}$. Then by proceeding as we did for $\mathcal{F}$, we arrive at an integral formula and the following properties for $\mathcal{G}$ :

- $\mathcal{G} \mathcal{T}_{\alpha} f=\mathcal{R}_{-\alpha} \mathcal{G} f$,

- $\mathcal{G} \mathcal{D}_{\lambda} f=\frac{1}{|\lambda|} \mathcal{D}_{\frac{1}{\lambda}} \mathcal{G} f$

- $\mathcal{G} \mathcal{R}_{\alpha} f=\mathcal{T}_{\alpha} \mathcal{G} f$.

We examine $\mathcal{I}=\mathcal{F} \mathcal{G}$ and how it interacts with $\mathcal{T}_{n}, \mathcal{D}_{\lambda}$ and $\chi_{[0,1)}$. Using the algebraic properties of $\mathcal{F}$ and $\mathcal{G}$ :

$$
\begin{gathered}
\mathcal{I} \mathcal{T}_{n} f=\mathcal{F} \mathcal{G} \mathcal{T}_{n} f=\mathcal{F} \mathcal{R}_{-n} \mathcal{G} f=\mathcal{T}_{n} \mathcal{F} \mathcal{G} f=\mathcal{T}_{n} \mathcal{I} f \\
\mathcal{I D}_{\lambda} f=\mathcal{F} \mathcal{G} \mathcal{D}_{\lambda} f=\frac{1}{|\lambda|} \mathcal{F} \mathcal{D}_{\frac{1}{\lambda}} \mathcal{G} f=\frac{|\lambda|}{|\lambda|} \mathcal{D}_{\lambda} \mathcal{F} \mathcal{G} f=\mathcal{D}_{\lambda} \mathcal{I} f
\end{gathered}
$$


Thus $\mathcal{I}$ commutes with $\mathcal{T}_{n}$ and $\mathcal{D}_{\lambda}$, so if we can determine the image of $\chi_{[0,1)}$ we can determine the image of $D$. Using the integral formula and a little contour integration we see:

$$
\begin{aligned}
\left(\mathcal{I} \chi_{[0,1)}\right)(x)=\left(\mathcal{F} \mathcal{G} \chi_{[0,1)}\right)(x) & \\
& =\mathcal{F}\left(\frac{e^{i \omega}-1}{i \omega}\right) \\
& =\int \frac{e^{i \omega}-1}{i \omega} e^{-i \omega x} d \omega=2 \pi \chi_{[0,1)}(x)
\end{aligned}
$$

for almost every $x$. So $\mathcal{I}$ acts on $D$ by multiplying by $2 \pi$. Using the fact that $\mathcal{I}$ is continuous we see that $\mathcal{I}$ acts on all of $L^{2}(\mathbb{R})$ in this way, and so $(2 \pi)^{-1} \mathcal{G}$ is a right inverse for $\mathcal{F}$. Naturally a similar argument shows that it is also a left inverse.

\section{To finish up}

This is a curious construction of the Fourier transform. It is even quite easy to extend it to $L^{2}\left(\mathbb{R}^{n}\right)$. One interesting point I didn't touch on is that we may change the first rule with which we defined $\mathcal{F}$ from:

- for the characteristic function of $[0,1)$ we define

$$
\mathcal{F}\left(\chi_{[0,1)}\right)=\frac{1-e^{-i \omega}}{i \omega}
$$

to the seemingly weaker:

- $\mathcal{F}\left(\chi_{[0,1)}\right)$ is continuous at zero and has value 1 at zero.

This is because $\chi_{[0,1)}$ satisfies the dilation equation:

$$
\chi_{[0,1)}(x)=\chi_{[0,1)}(2 x)+\chi_{[0,1)}(2 x-1),
$$

but that is another story, [5].

\section{References}

[1] Elias M. Stein and Guido Weiss, Introduction to Fourier Analysis on Euclidean Spaces. Princeton University Press: 1971. 
[2] H. Dym and H. P. McKean, Fourier Series and Integrals. Academic Press: 1972.

[3] Yves Meyer, Wavelets and Operators. Cambridge University Press: 1992.

[4] Ingrid Daubechies, Ten Lectures on Wavelets. SIAM: (1992).

[5] David Malone, Fourier analysis, multiresolution analysis and dilation equations, Master's thesis (1997), University of Dublin.

David Malone

Department of Mathematics

Trinity College

Dublin 2

Ireland 